\title{
The value of SPECT/CT imaging of lacrimal glands as a means of assessing the activity of Graves' orbitopathy
}

\author{
Ru-Xuan Zhao', Ting-Ting Shi', Sha Luo ${ }^{2}$, Yun-Fu Liu ${ }^{3}$, Zhong Xin ${ }^{1}$ and Jin-Kui Yang(1)1 \\ 'Department of Endocrinology, Beijing Diabetes Institute, Beijing Tongren Hospital, Capital Medical University, Beijing, China \\ 2Department of Nuclear Medicine, Beijing Tongren Hospital, Capital Medical University, Beijing, China \\ ${ }^{3}$ Department of Radiology, Beijing Tongren Hospital, Capital Medical University, Beijing, China
}

Correspondence should be addressed to J-K Yang: jkyang@ccmu.edu.cn

\begin{abstract}
Background: Graves' orbitopathy (GO) is an autoimmune disease with mechanical impairment of orbital muscles and lacrimal gland dysfunction. The frequently used methods of assessing GO activity include Clinical Activity Score (CAS), CT, and MRI. These approaches are mainly associated with orbital muscles; however, there are not many studies that focus on the lacrimal gland inflammation of GO patients.

Objective: The aim of this study is to assess the usefulness of ${ }^{99 \mathrm{~m} T c-D T P A}$ single-photon emission (SPE) CT/CT in evaluating the lacrimal gland inflammation in GO, as compared with other methods.

Methods: A retrospective analysis of 48 patients with active GO compared with 33 controls was conducted. All subjects underwent clinical-endocrinological analyses, CAS evaluation, CT scans, and SPECT/CT examination. Lacrimal gland dimensions were determined and analyzed.

Results: The lacrimal glands in patients with $\mathrm{GO}$ were significantly larger in all measured dimensions $(P<0.001)$ on CT scans relative to those in controls. Increased lacrimal gland diethylene triamine pentaacetic acid (DTPA) uptake ratios $(P<0.001)$ were displayed in active GO patients compared to controls and were also correlated with thyrotropin receptor antibody levels. The cut-off value for discriminating active and inactive disease was calculated to be 1.735 , with specificity of $82.6 \%$ and sensitivity of $74.2 \%$.

SPECT/CT uptake ratios and CAS values were positively correlated in all GO patients. SPECT/CT uptake ratios were also positively correlated with CT measurements including lacrimal gland volume and coronal width in GO patients. Conclusions: These data indicated that lacrimal gland SPECT/CT images can serve as a good tool for assessing the inflammation and disease activity of GO.
\end{abstract}

\section{Introduction}

Graves' orbitopathy (GO) is an autoimmune disease which frequently arises in patients suffering from Graves' disease $(1,2)$. GO, often involving both eyes, is one of the most common orbital diseases in adults $(3,4)$. GO can cause exophthalmos and redness of the eyelids. In severe cases, it can also cause exposed keratitis, diplopia, compression of the optic nerve, and even blindness. As such, GO is a sightthreatening disease and can cause aesthetic damage and adversely affect the quality of life $(5,6,7)$. Between 65 and $85 \%$ of GO patients exhibit lacrimal gland involvement, which manifests as dryness, photophobia, tearing, and even corneal damage $(8,9)$. Some studies have shown https://ec.bioscientifica.com

https://doi.org/10.1530/EC-21-0590 (c) 2022 The authors Published by Bioscientifica Ltd

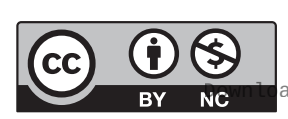

This work is licensed under a Creative Commons Attribution-NonCommercial 4.0 International License. ded from Bioscientifica.com at 04/26/2023 02:20:11PM 
that lacrimal glands in GO patients exhibit inflammation attributable to both autoimmune and inflammatory sources $(10,11)$. Lacrimal gland acinar cells can express thyroid-stimulating hormone receptors (12) and exhibit multifocal infiltration by lymphocytes as well as clear adipose tissue hyperplasia (13). This further confirms that the lacrimal gland is the target organ associated with symptoms in GO patients and indicates that evaluating the lacrimal gland inflammation may be of clinical relevance.

At present, GO is diagnosed based on a combination of clinical symptoms, imaging, and laboratory examinations. Accurate assessment of GO severity and clinical activity in affected patients is essential in order to determine optimal follow-up treatment strategies (3). Disease activity is measured based upon clinical activity score (CAS) values (14), with a CAS $\geq 3 / 7$ being indicative of active GO. However, CAS score is subjective and mostly cliniciandepended. Some prior studies have used CT scans and MRI in measuring lacrimal gland herniation as well as inflammation to assist thyroid associated ophthalmopathy (TAO) activity staging. The size and density of lacrimal glands in patients with $\mathrm{GO}$ are increased in relation to healthy individuals without this disease upon CT and MRI imaging $(15,16,17,18)$. However, CT scans and MRI are usually applied for determining the changes in morphology, not the severity. Advances in nuclear medicine enable simultaneous anatomical and functional evaluations of tissues. Diethylene triamine pentaacetic acid (DTPA) is often employed as a tracer molecule in functional imaging contexts, as it can aid in the detection of inflammation owing to its increased ability to pass through capillary walls in inflamed retrobulbar or lacrimal gland tissues in those with active GO (19). Single-photon emission CT (SPECT)/CT utilizes this tracer in combination with CT imaging to facilitate the reconstruction of 3D images of sites of inflammation, thus offering spatial insights regarding biological activity in the examined area (20). At present, ${ }^{99 \mathrm{~m} T c-D T P A}$ SPECT/CT imaging is rarely reported in the evaluation of lacrimal gland inflammation. Herein, we combined SPECT/CT and CT scans in measuring the inflammation of GO to evaluate the role of SPECT/CT as a means of assessing disease activity and severity of patients with active GO.

Using a SPECT/CT approach, in the present study, we assessed lacrimal gland uptake ratios in GO patients and controls and evaluated correlations between these ratios and more traditional disease severity and activity parameters derived from CT or CAS analyses. The goal of this approach was to explore the validity of lacrimal gland SPECT/CT imaging results as a means of evaluating inflammatory severity and activity in patients with active GO.

\section{Methods}

\section{Study subjects}

For this study, we retrospectively analyzed a total of 48 patients with active GO (16 men, 32 women; aged: 18-69 years, mean: $46.58 \pm 10.9$ ) as well as 33 patients with inactive GO (10 men, 23 women; aged: 21-65 years, mean: $44.62 \pm 9.6)$. All of these patients underwent both CT scanning and ${ }^{99 \mathrm{~m}} \mathrm{Tc}-\mathrm{DTPA}$ orbital SPECT/CT within 10 days between August 2017 and September 2019 at Beijing Tongren Hospital. In active GO group, 24 patients were considered to be hyperthyroid and another 24 to be euthyroid at the time of assessment, and only patients in the former category were treated with antithyroid drugs. Thyroid function in the control group matched that in the active GO group. We excluded all patients treated with steroids, thyroid surgery, or radiotherapy from the present study. Patients were diagnosed with GO based upon European Group on Graves' Orbitopathy (EUGOGO) Guidelines, with GO CAS scores being evaluated with a 7 point-scale as per EUGOGO recommendations (14). For this scale, patients were evaluated for the following symptoms: spontaneous retrobulbar pain, pain on attempted upward or downward gaze, eyelid redness, conjunctival redness, caruncle or plica swelling, eyelid swelling, and conjunctival swelling (chemosis), with 1 point awarded per symptom. A CAS value of 3 or higher is consistent with active GO disease. These criteria can also be used to categorize GO disease severity as being either mild, moderate-to-severe, or sight-threatening according to NOSPECS. No included patients had any history of ocular trauma, orbital tumors, or any other known diseases affecting the lacrimal glands.

The study was approved by Beijing Tongren Hospital, Capital Medical University. Consent was obtained from each patient after a full explanation of the purpose and nature of all study procedures.

The characteristics of all subjects, including age, gender, current treatments, medical history, history of drug use, and history of smoking were recorded. Clinical data including serum free triiodothyronine 3 (FT3), free triiodothyronine 4 (FT4), thyroid-stimulating hormone (TSH), and thyrotropin receptor antibody (TRAb) levels were measured to assess 
thyroid function. Schirmer's test with anesthesia (STA) was performed on the day of CAS evaluation (21). Study patient characteristics are shown in Table 1.

\section{CT scans}

A 64 Spiral CT Scan Instrument (Philips Brilliance) was used for all orbital CT scanning, based upon a 2-mm slice thickness orbit protocol as in previous reports (22), with a Philips image post-processing workstation (extended Brilliance workspace, EBW) being used for image viewing and reconstruction. As it was challenging to distinguish between the orbital and palpebral lobes of the lacrimal gland in these images, the entirety of the gland was treated as a single structure, with the image in which the gland was largest being selected for analysis. An outline of the lacrimal gland was drawn using axial images (volume). We then determined the width and length of the gland in these axial images, measuring from the most posterior tip to the most anterior tip (AL) and measuring the widest distance from the lateral edge to the medial edge of the gland perpendicular to AL (AW) (Fig. 1A and B). Comparable measurements were then made in coronal images, determining the longest dimension from the superior tip of the lacrimal gland to the inferior tip (CL) and then measuring the widest dimension from the lateral edge to the medial edge perpendicular to CL (CW) (Fig. 1C and D). This analysis was conducted both for the axial and coronal images in which the gland appeared largest, as well as in the images before and after the selected image, with these three values being averaged together to calculate the final value. We also ensured that the maximum diameter of the bilateral lens and the inner segment of the optic nerve was present on the same side. We then connected the outer edges of the orbit, measuring the vertical distance from the most anterior tip of the lacrimal gland to the line as a means of determining the degree of lacrimal gland prolapse (Fig. $1 \mathrm{~A}$ and $\mathrm{B})$. All measurements were recorded in millimeters.
All orbital CT scans were given to three clinicians including an experienced radiologist, an endocrinologist, and a fifth-year intern for analysis, and to one observer on consecutively 3 days. The intraclass correlation coefficient (ICC) was applied to evaluate the inter-observer variation among observers for measuring the lacrimal gland parameters in CT images. All three observers independently measured CT data from ten random selected images and reconstructed and calculated lacrimal gland parameters. The coefficient of variation (CV) was applied to evaluate the intra-observer variation of one observer for repeated estimations. CT data of one patient with typical TAO were used to calculate lacrimal gland parameters over 3 consecutive days by the same observer.

\section{SPECT/CT}

A total of $740 \mathrm{MBq}$ of radiotracer ${ }^{99 \mathrm{~m}} \mathrm{Tc}-\mathrm{DTPA}$ (High Tech Atomic Co., Ltd., Beijing, China; >95\% purity) were intravenously injected into subjects, who then underwent orbital SPECT/CT (United States GE Discovery NM670) data acquisition $20 \mathrm{~min}$ post-injection. The following parameters were used for CT scanning: $120 \mathrm{kV}, 60 \mathrm{~mA}$, $2.5 \mathrm{~mm}$ slice thickness. The following parameters were used for SPECT imaging: $128 \times 128$ matrix, 6\% frame, $30 \mathrm{~s} /$ frame, 60 total frames. The GE xeleris 3.0 workstation software was used to fuse the SPECT and CT images.

Using region of interest (ROI) technology, lacrimal glands (R1) were outlined in the three consecutive axial and coronal images exhibiting maximal tracer uptake. On the optimal axial image, ROIs corresponding to the occipital lobe region of the brain (R2) and the occipital region (R3) were drawn as references, avoiding the nasopharynx. All ROIs were exactly 13 pixels in size, and the maximal and mean counts were measured for these ROIs as R1(max), R1(avg), R2, and R3. We then calculated the following uptake ratios: $\mathrm{R} 1(\max ) / \mathrm{R} 2, \mathrm{R} 1(\mathrm{avg}) / \mathrm{R} 2, \mathrm{R} 1(\max ) / \mathrm{R} 3$, and R1(avg)/R3 (Fig. 2A and B).

Table 1 Clinical characteristics of inactive GO and active GO patients.

Number of patients $(n)$
Sex (male/female)
Age (years)
Smokers $(n, \%)$
FT3 (pmol/L, median; reference range, 3.50-6.50)
FT4 (pmol/L, median; reference range, 11.50-22.70)
TSH (uIU/mL, median; reference range, $0.55-4.78)$
TRAb (IU/L, median; reference range, $0.00-1.75)$
Sum of tearing (mm)

\begin{tabular}{c}
\hline Inactive GO \\
\hline 33 \\
$10 / 23$ \\
$44.62 \pm 9.6$ \\
$7(21.2)$ \\
$4.58(3.51-7.37)$ \\
$14.68(11.79-24.18)$ \\
$1.14(0.001-4.72)$ \\
$2.42(0.50-13.60)$ \\
$17(5-30)$
\end{tabular}

\begin{tabular}{c}
\hline Active GO \\
\hline 48 \\
$16 / 32$ \\
$46.58 \pm 10.9$ \\
$19(29.6)$ \\
$5.16(3.11-7.28)$ \\
$16.64(11.98-23.84)$ \\
$0.48(0.001-2.12)$ \\
$5.51(1.33-40.00)$ \\
$16(2-30)$
\end{tabular}

\begin{tabular}{c}
\hline $\boldsymbol{P}$ \\
\hline 0.782 \\
0.615 \\
0.233 \\
0.581 \\
0.454 \\
0.215 \\
0.001 \\
0.371
\end{tabular}

FT3, free triiodothyronine 3; FT4, free triiodothyronine 4; TSH, thyroid-stimulating hormone, TRAb, thyrotropin receptor antibody.

$\begin{array}{lr}\text { https://ec.bioscientifica.com } & \text { C } 2022 \text { The authors } \\ \text { https://doi.org/10.1530/EC-21-0590 } & \text { Published by Bioscientifica Ltd }\end{array}$

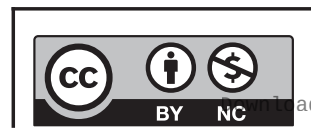

This work is licensed under a Creative Commons Attribution-NonCommercial 4.0 International License. ded from Bioscientifica.com at 04/26/2023 02:20:11PM 

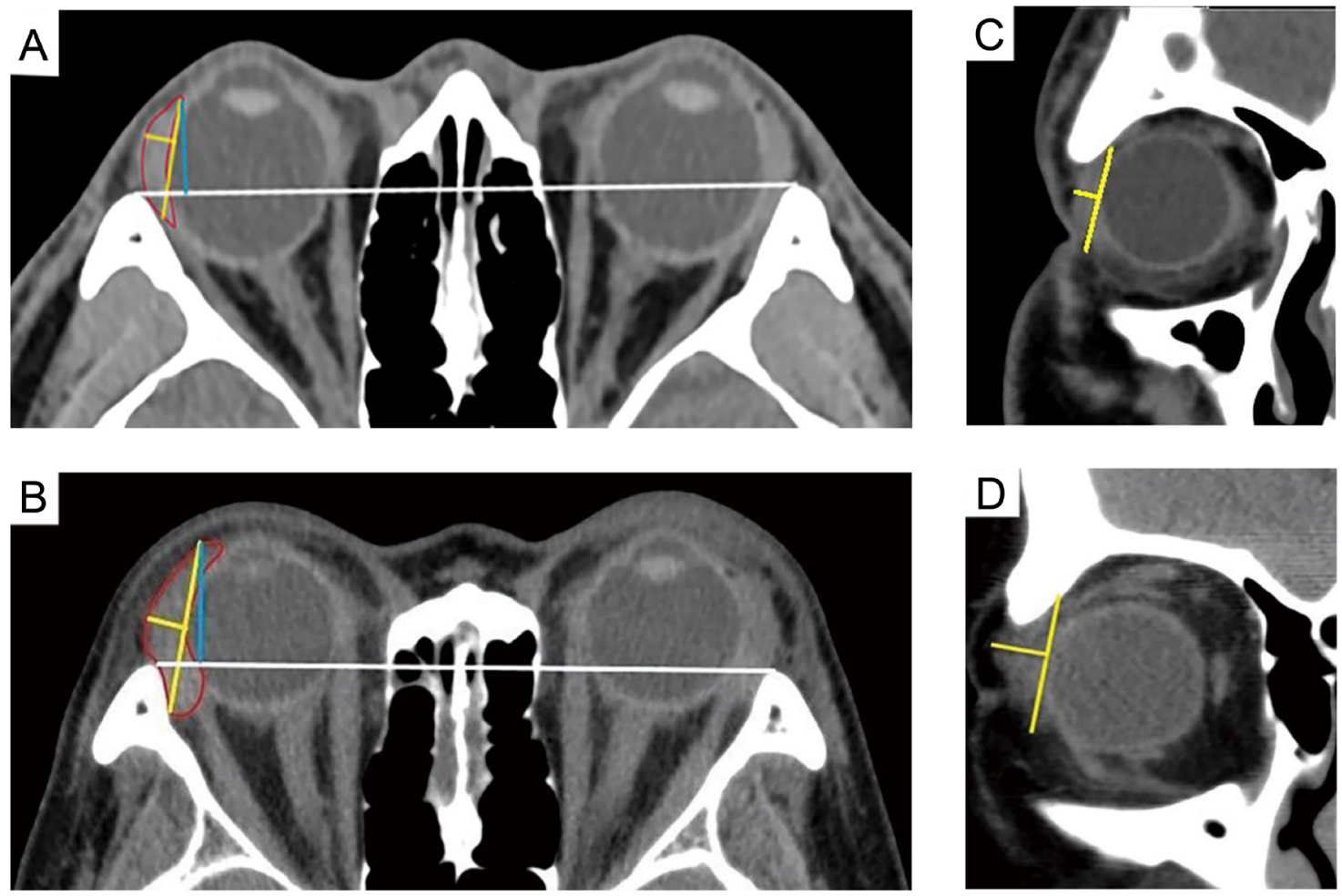

$E$

Volume

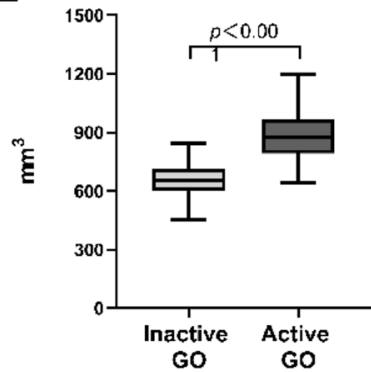

Axial Width

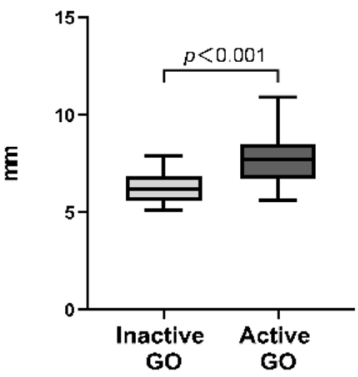

Prolapse

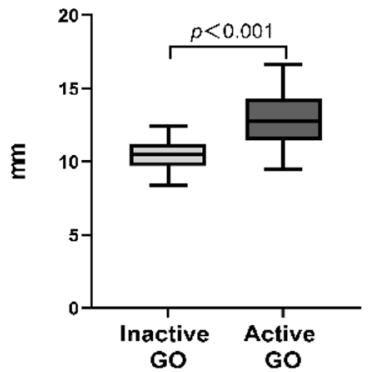

Coronal Length

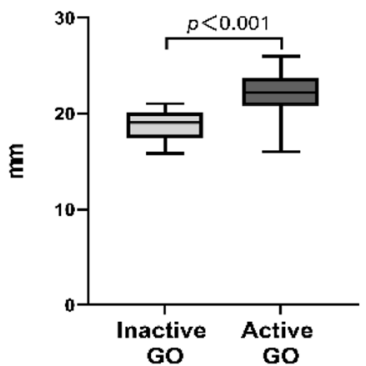

Axial Length

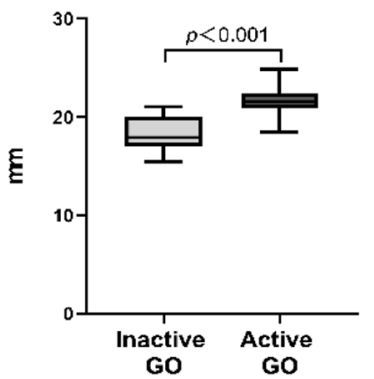

Coronal Width

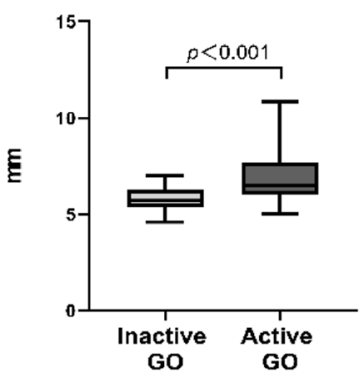

Figure 1

Quantitative measurements and analysis of lacrimal gland on CT scans in inactive GO and active GO patients. (A and B) Volume (red), prolapse (blue), length, and width (yellow) of lacrimal gland in inactive and active GO patients on axial CT images. (C and D) Length and width (yellow) of lacrimal gland in inactive and active GO patients on coronal CT images. (E) Comparison of quantitative dimensions of lacrimal gland in inactive and active GO patients.

https://ec.bioscientifica.com https://doi.org/10.1530/EC-21-0590 (c) 2022 The authors Published by Bioscientifica Ltd

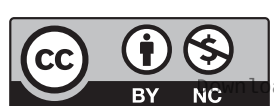

This work is licensed under a Creative Commons Attribution-NonCommercial 4.0 International License. ded from Bioscientifica.com at 04/26/2023 02:20:11PM 

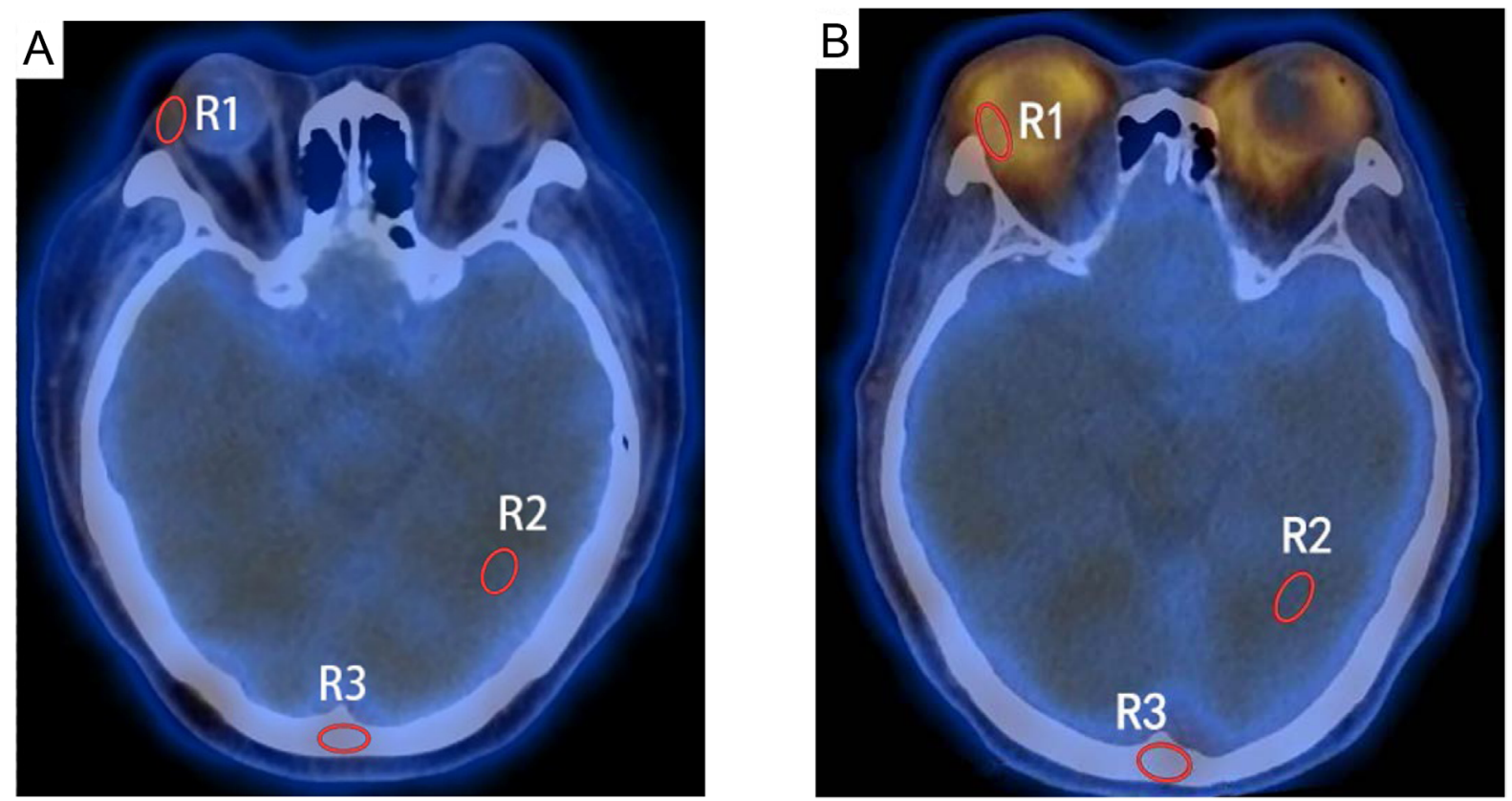

C
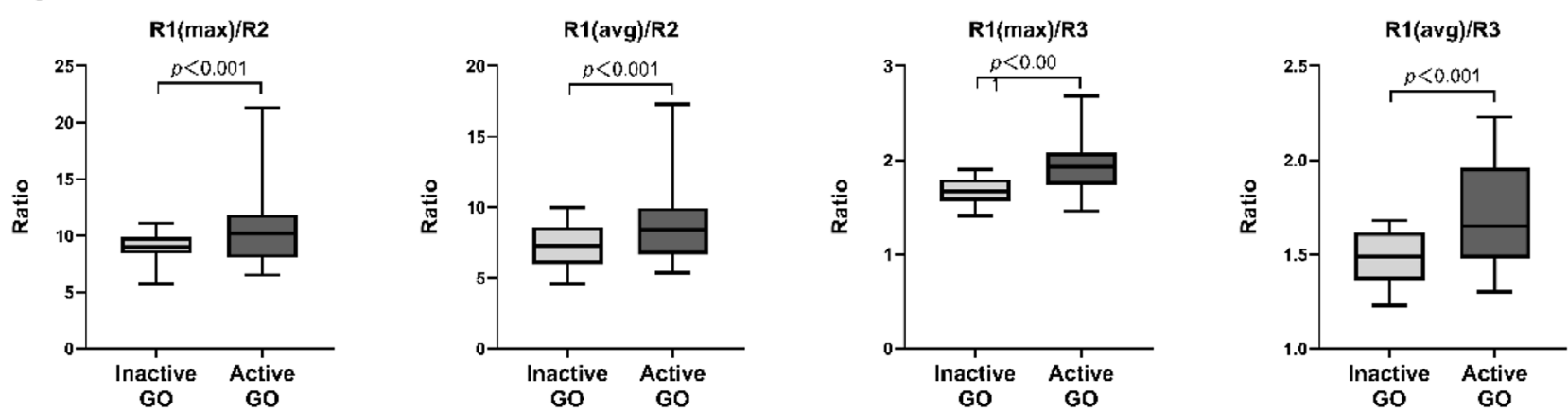

Figure 2

Measurements and analysis of lacrimal gland on SPECT/CT in inactive GO and active GO patients. (A and B) The outlined region of interests (R1: lacrimal gland, R2: occipital lobe brain region, R3: occipital region) on SPECT/CT images in inactive and GO patients. (C) Comparison of four semi-quantitative indexes (R1(max)/R2, R1(avg)/R2, R1(max)/R3, R1(avg)/R3) between inactive and GO patients.

The above image analysis is performed by two nuclear medicine physicians. Two physicians measured the quantitative parameters of SPECT/CT lacrimal gland of three randomly selected subjects. The ICC $>0.97$ indicated good reproducibility.

\section{Statistical analysis}

SPSS v25.0 (Armonk, NY, USA) was used for all analyses in the present study. The Shapiro-Wilks test was used to assess the normality of datasets, with Student's $t$-tests and MannWhitney $U$ tests being used to compare normally and non-normally distributed data, respectively. Spearman correlation tests were used to assess correlations between continuous variables. Diagnostic accuracy was evaluated using receiver operating characteristic (ROC) curve analysis. $P<0.05$ was the significance threshold for this study.

\section{Results}

\section{Demographic features}

We examined outcomes in 48 active GO and 33 inactive GO patients in the present study. There were no significant differences in age $(P=0.615)$ or gender $(P=0.782)$ between these cohorts. There was also a similar number of smokers in both groups $(P=0.233)$. Control patients exhibited thyrotropin receptor autoantibody (TRAb) as $2.42 \mathrm{IU} / \mathrm{L}$, whereas active GO patients had significantly 
higher TRAb levels as $5.51 \mathrm{IU} / \mathrm{L}(P<0.001)$. There were no significant differences in thyroid function (FT3, FT4, and TSH) between controls and active GO patients $(P=0.581$, $P=0.454, P=0.215)$. The sum of tearing did not differ significantly between two groups $(P=0.371)$ (Table 1$)$.

\section{CT dimensions}

Control patients exhibited a mean lacrimal gland volume of $654.48 \mathrm{~mm}^{3}$ (SD, 95.16), while this volume was significantly larger in active GO patients at $888.04 \mathrm{~mm}^{3}$ (SD, 129.34; $P<0.001$ ) (Fig. 1E). Control patients exhibited an average $10.47 \mathrm{~mm}$ (SD, 0.95) lacrimal gland prolapse, whereas this value was significantly higher at $12.78 \mathrm{~mm}$ (SD, 1.80) in GO patients $(P<0.001)$ (Fig. 1E). Control patients exhibited a mean lacrimal gland axial length of $18.28 \mathrm{~mm}(\mathrm{SD}, 1.59)$, whereas in GO patients this value was $21.53 \mathrm{~mm}$ (SD, 1.47) (Fig. 1E). Controls had a mean axial width of $6.28 \mathrm{~mm}$ (SD, 0.77), vs $7.77 \mathrm{~mm}(\mathrm{SD}, 1.42)$ in GO patients (Fig. 1E). Control patients had a mean coronal length of $18.92 \mathrm{~mm}(\mathrm{SD}, 1.40)$ vs $22.31 \mathrm{~mm}(\mathrm{SD}, 1.95)$ in GO patients (Fig. 1E). Control patients had a mean coronal width of $5.81 \mathrm{~mm}(\mathrm{SD}, 0.59)$ vs $6.90 \mathrm{~mm}(\mathrm{SD}, 1.29)$ in GO patients (Fig. 1E). The lacrimal gland axial length, axial width, coronal length, and coronal width in GO patients were all significantly elevated relative to control patients $(P<0.001)$ (Fig. 1E).

The ICC values for lacrimal gland volume, prolapse, axial length, axial width, coronal length, and coronal width for three observers were $0.905,0.923,0.951,0.934$, 0.910 , and 0.911 , respectively. The CV values for these measurements were all below $5 \%$.

\section{SPECT/CT}

We observed a clear increase in DTPA uptake in the lacrimal glands of active GO patients relative to controls. Using the uptake ratio for the occipital lobe of the brain as a correction factor, the maximum $(\mathrm{R} 1(\max ) / \mathrm{R} 2)$ and average (R1(avg)/R2) lacrimal gland uptake count in active GO patients were $10.36 \pm 2.88$ and $8.71 \pm 2.35$, respectively. In contrast, in controls, these values were same $9.03 \pm 1.06$ and $7.38 \pm 1.48$, respectively, with significant differences in both of these uptake ratios being detected between active GO patients and controls (both $P<0.001$; Fig. 2C). Based on the occipital ${ }^{99 \mathrm{~m} T c-D T P A}$ uptake rate, the maximum (R1max/R3) and average (R1avg/R3) lacrimal gland uptake count ratios were $1.93 \pm 0.26$ and $1.69 \pm 0.23$, respectively, whereas in control patients these ratios were $1.67 \pm 0.15$ and $1.48 \pm 0.14$, respectively. Thus, there were thus significant differences in both of these ratios between

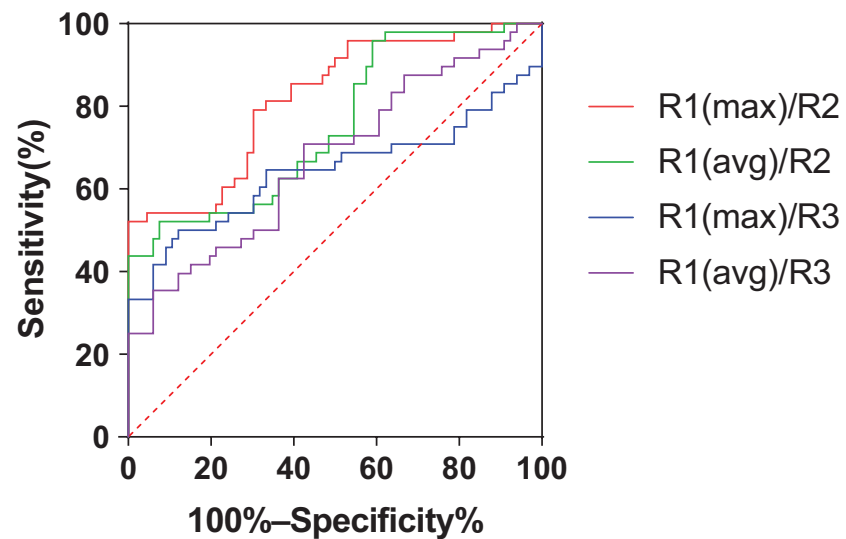

Figure 3

Receiver operating characteristic (ROC) curve analysis of lacrimal gland DTPA uptake. (R1, lacrimal gland; R2, occipital lobe brain region; R3, occipital region).

controls and active GO patients (both $P<0.001$; Fig. 2C). When using the occipital lobe of the brain or the occipital ${ }^{99 \mathrm{~m}}$ Tc-DTPA uptake rate as a correction factor, we were thus able to determine that lacrimal gland uptake ratios in active GO patients were higher than in controls, consistent with results from visual analyses. ROC curve tests of 4 uptake radios were assessed using the area under the ROC curve as an index performance (Fig. 3). ROC curve-based best threshold value between active vs inactive disease was defined as the cut-off value. Optimal diagnostic performance was shown in $\mathrm{R} 1(\max ) / \mathrm{R} 2$. The cut-off value for discriminating active and inactive disease was calculated to be 1.735 , with an area under curve (AUC) of 0.86 , specificity of $82.6 \%$, and sensitivity of $74.2 \%$.

\section{Correlation analyses}

We next assessed correlations between SPECT/CT findings and other clinical findings in these same patients. We found that the lacrimal gland/occipital ${ }^{99 \mathrm{~m}} \mathrm{Tc}$-DTPA uptake ratio in active GO patients was correlated with TRAB $(\mathrm{R} 1(\max ) / \mathrm{R} 3 \mathrm{r}=-0.389 P=0.005, \mathrm{R} 1$ (avg)/R3 $\mathrm{r}=-0.392$ $P=0.003)$, whereas that in control groups was not $(P>0.05)$. The lacrimal gland/occipital lobe of the brain ${ }^{99 \mathrm{~m}}$ Tc-DTPA uptake ratio was not correlated with TRAB $(P>0.05)$. Neither of these ratios were correlated with qualitative indices, smoking, or the sum of tearing $(P>0.05)$.

We further used a Spearman rank correlation analysis approach to compare the relationship between CAS scores and SPECT/CT results in two groups. This revealed that the lacrimal gland/occipital lobe brain maximum and average uptake count ratios were positively correlated with CAS in patients with GO (Fig. 4; $\mathrm{r}=0.688, P=0.03$;

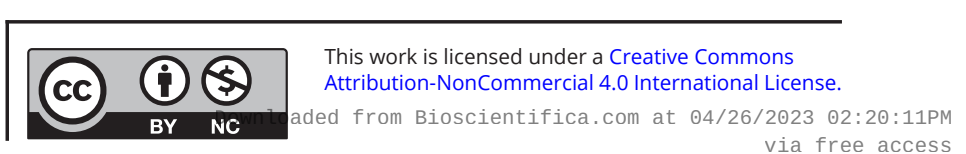


A

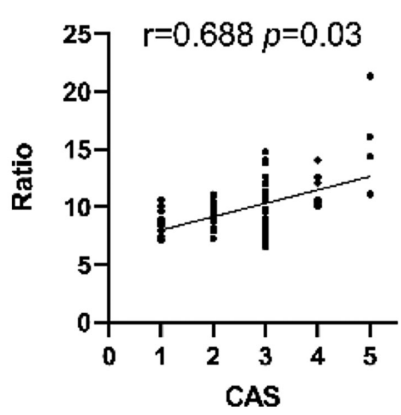

B

R1(avg)/R2

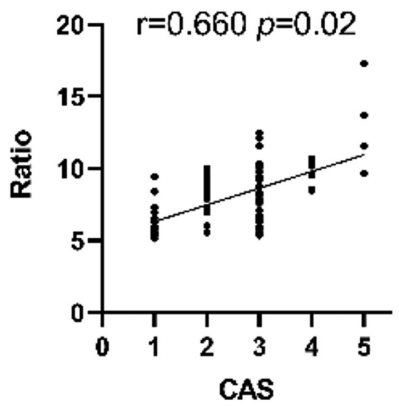

C

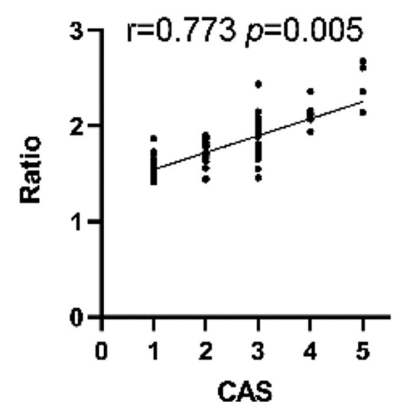

D

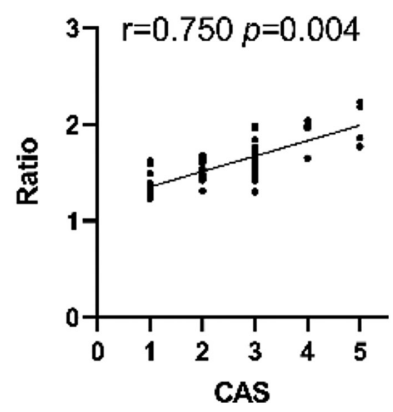

Figure 4

Correlation analysis between region of interests (ROIs) of lacrimal gland on SPECT/CT images and CAS in GO patients.

$\mathrm{r}=0.660, P=0.02)$. In addition, both lacrimal gland/ occipital maximum and average uptake count ratios were correlated with CAS values (Fig. $4 ; \mathrm{r}=0.773, P=0.005$; $\mathrm{r}=0.750, P=0.004)$.

We further found that, when comparing CT dimensions and SPECT/CT results in active GO patients, , lacrimal gland/occipital lobe maximum and average uptake count ratios (R1(max)/R2, R1(avg)/R2, R1(max)/R3, and R1(avg)/ R3) were positively correlated with lacrimal gland volume (Table 2; $\mathrm{r}=0.405 P=0.005, \mathrm{r}=0.448 P=0.001, \mathrm{r}=0.440$, $P=0.002, \mathrm{r}=0.414, P=0.003)$ as measured via CT. In addition, we detected correlations between ${ }^{99 m}$ Tc-DTPA lacrimal gland/occipital lobe maximum or average uptake count ratios and coronal width (Table $2 ; \mathrm{r}=0.307 P=0.040$, $\mathrm{r}=0.302 P=0.037)$ as measured via CT. In addition, lacrimal gland/occipital maximum and average uptake count ratios were also correlated with coronal width on CT images (Table $2 ; \mathrm{r}=0.304 P=0.048, \mathrm{r}=0.392 P=0.006$ ). We did not observe any correlations between the SPECT/CT imaging results and other CT dimensions such as prolapse, axial length, axial width, or coronal length. No significant correlations were observed between CT and CAS findings, with a correlation between lacrimal gland volume and CAS

Table 2 Correlation analysis between region of interests (ROIs) of lacrimal gland on SPECT/CT images and CT dimensions in active $\mathrm{GO}$ patients.

\begin{tabular}{|c|c|c|c|c|}
\hline & \multicolumn{2}{|c|}{ Volume } & \multicolumn{2}{|c|}{ Coronal width } \\
\hline & $r$ & $P$ & $r$ & $P$ \\
\hline $\mathrm{R} 1(\max ) / \mathrm{R} 2$ & 0.405 & 0.005 & 0.307 & 0.040 \\
\hline R1(avg)/R2 & 0.448 & 0.001 & 0.302 & 0.037 \\
\hline R1(max)/R3 & 0.440 & 0.002 & 0.304 & 0.048 \\
\hline R1(avg)/R3 & 0.414 & 0.003 & 0.392 & 0.006 \\
\hline
\end{tabular}

R1, lacrimal glands; R2, the occipital lobe region of the brain; R3, the occipital region. of $0.208(P=0.155)$ and a correlation between lacrimal gland coronal width and CAS of $0.233(P=0.111)$.

\section{Discussion}

GO is among the most common causes of autoimmune orbitopathy, with moderate-to-severe GO having the potential to induce corneal and optic nerve damage, potentially resulting in blindness (23). It is therefore important that means of detecting and evaluating clinical stage and inflammatory activity be identified in order to choose appropriate treatments for GO patients. For active GO patients, glucocorticoids (GCs) or immunosuppressive therapy may be needed, but in case GO is judged to be inactive, chances of a positive response to GCs and immunosuppression are small. However, it is often difficult to judge whether GO is active or inactive. In the present study, we employed a SPECT/CT image fusion strategy, revealing that DTPA uptake rate in active GO group was much higher than inactive GO group and correlated with TRAB level. There was correlation between lacrimal gland SPECT/CT imaging results and both CAS values as well as lacrimal gland CT measurements in GO patients. We showed that the lacrimal gland DTPA uptake rate may be a good parameter to distinguish between inactive and active GO, which may help improve the diagnostic ability of inflammatory activity in some clinical cases.

GO-related changes in lacrimal glands are associated with uneven tear distributions on the ocular surface, shortened tear film rupture time, accelerated tear evaporation, and increased tear osmotic pressure, which in turn lead to decreased lacrimal secretory function $(15,24$, $25,26)$. GO progression is associated with autoantibody and inflammatory mediator activity. Lacrimal acinar cells express TSHR (12), and the expression of ICAM-1 in lacrimal

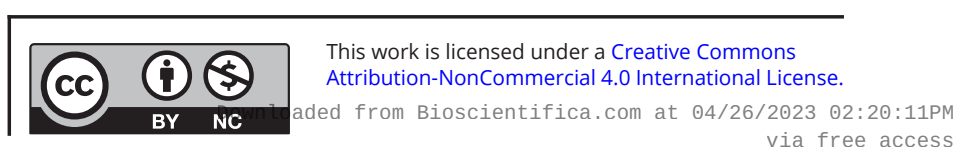


gland epithelial cells and lymphocytes in GO patients is significantly elevated in relation to the normal control group (13). A study of cytokines and chemokines in tears from $21 \mathrm{GO}$ patients and 10 healthy controls found that interleukin (IL)-1 $\beta$, IL-6, and IL-8 levels were significantly higher in those with active $\mathrm{GO}$ in relation to patients with inactive GO and healthy controls (6). Capillary permeability around the lacrimal glands is also increased in GO patients due to disease-related inflammation. The DTPA tracer molecule can be used to detect inflammation owing to its increased ability to pass through capillary walls in inflamed tissues $(19,27,28)$. One study found a significant difference in lacrimal gland uptake ratios between GO patients and healthy individuals, with these lacrimal gland uptake ratios being positively correlated with CAS values in GO patients (29). In this study, we found that when using brain or occipital bone as reference tissues, our results based on active GO patients and inactive GO patients were consistent with previous results. The best threshold $\mathrm{R} 1$ (max)/R2 ratio for discriminating active and inactive disease was calculated to be 1.735 , with specificity of $82.6 \%$ and sensitivity of $74.2 \%$. However, the results need to be verified by observing reaction of immune suppressive therapy in active GO patients classified by this ratio. We will fix it in future work. CT is a diagnostic imaging approach that is frequently employed in GO patients, and as such, these scans can accurately define lacrimal gland boundaries, allowing for measurements of gland size and morphology in an objective manner based upon lacrimal gland volume, coronal length, coronal width, axial length, and axial width $(20,25,29)$. Quantitative measurements of CT-derived lacrimal gland parameters can offer insight into disease severity based upon tissue responses to local inflammation. Indeed, we observed that GO patients exhibited CT lacrimal gland parameters that were significantly different from those in control patients, as in many previous studies $(25,29,30)$. We also evaluated the relevance of a novel lacrimal gland parameter, lacrimal gland prolapse, which was measured based on exophthalmos. We found that such prolapse was significantly increased in GO patients. This was in line with the other measured parameters, suggesting that such a measurement may be a valuable means of describing lacrimal gland frontier prolapse on CT images. Indeed, in the present report, we found that SPECT/CT lacrimal gland uptake ratios were correlated with the volume and coronal width of the lacrimal gland as measured by CT, suggesting that SPECT/CT is also an effective means of detecting lacrimal gland morphological changes. However, the correlation between SPECT/CT and axial width was poor. This may be due to measurement inaccuracies as a consequence of eyelids blocking a portion of the widened lacrimal gland.

This study has certain limitations. For one, our sample size was small, and thus, future larger studies are needed. In addition, this was a single-center study, and as such, future multicenter analyses are warranted. Furthermore, we did not utilize evenly distributed patients with moderate-to-severe GO, with more patients having a CAS value of 3 and fewer having a CAS value of 5 in the present study cohort. Whether lacrimal gland and retrobulbar changes occur in parallel with one another also remains to be assessed.

In conclusion, ${ }^{99 \mathrm{~m}} \mathrm{Tc}-\mathrm{DTPA}$ SPECT/CT of the lacrimal gland is a means of visually integrating anatomical and functional metabolic data. This approach offers insights into changes in both lacrimal gland morphology and tracer uptake, making it conducive to the clinical evaluation of GO disease severity and activity, offering an objective basis for patient treatment planning.

\section{Declaration of interest}

The authors declare that there is no conflict of interest that could be perceived as prejudicing the impartiality of the research reported.

\section{Funding}

This work did not receive any specific grant from any funding agency in the public, commercial, or not-for-profit sector.

\section{References}

1 Drui D, Du Pasquier Fediaevski L, Vignal Clermont C \& Daumerie C. Graves' orbitopathy: diagnosis and treatment. Annales d'Endocrinologie 201879 656-664. (https://doi.org/10.1016/j.ando.2018.08.005)

2 Kahaly GJ, Bartalena L, Hegedüs L, Leenhardt L, Poppe K \& Pearce SH. European Thyroid Association guideline for the management of graves' hyperthyroidism. European Thyroid Journal 20187 167-186. (https://doi.org/10.1159/000490384)

3 Bartalena L, Baldeschi L, Boboridis K, Eckstein A, Kahaly GJ, Marcocci C, Perros P, Salvi M \& Wiersinga WM. The 2016 European Thyroid Association/European Group on Graves' orbitopathy guidelines for the management of Graves' orbitopathy. European Thyroid Journal 20165 9-26. (https://doi.org/10.1159/000443828)

4 Lee HBH, Rodgers IR \& Woog JJ. Evaluation and management of Graves' orbitopathy. Otolaryngologic Clinics of North America 200639 923-942, vi. (https://doi.org/10.1016/j.otc.2006.07.008)

5 Park JJ, Sullivan TJ, Mortimer RH, Wagenaar M \& Perry-Keene DA. Assessing quality of life in Australian patients with Graves' ophthalmopathy. British Journal of Ophthalmology 200488 75-78. (https://doi.org/10.1136/bjo.88.1.75)

6 Wickwar S, McBain HB, Ezra DG, Hirani SP, Rose GE \& Newman SP. What are the psychosocial outcomes of treatment for thyroid eye disease? A systematic review. Thyroid 201424 1407-1418. (https://doi. $\operatorname{org} / 10.1089 /$ thy.2014.0037)

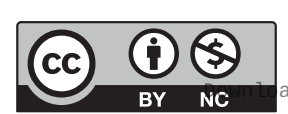

This work is licensed under a Creative Commons Attribution-NonCommercial 4.0 International License. Atrom Bioscientifica com at 04/26/2023 02:20:11PM 
7 Coulter I, Frewin S, Krassas GE \& Perros P. Psychological implications of Graves' orbitopathy. European Journal of Endocrinology 2007157 127-131. (https://doi.org/10.1530/EJE-07-0205)

8 Selter JH, Gire AI \& Sikder S. The relationship between Graves' ophthalmopathy and dry eye syndrome. Clinical Ophthalmology 2015 9 57-62. (https://doi.org/10.2147/OPTH.S76583)

9 Inoue S, Kawashima M, Arita R, Kozaki A \& Tsubota K. Investigation of Meibomian gland function and dry eye disease in patients with Graves' ophthalmopathy. Journal of Clinical Medicine 202092814. (https://doi.org/10.3390/jcm9092814)

10 Matheis N, Okrojek R, Grus FH \& Kahaly GJ. Proteomics of tear fluid in thyroid-associated orbitopathy. Thyroid 201222 1039-1045. (https:// doi.org/10.1089/thy.2012.0119)

11 Huang D, Xu N, Song Y, Wang P \& Yang H. Inflammatory cytokine profiles in the tears of thyroid-associated ophthalmopathy. Graefe's Archive for Clinical and Experimental Ophthalmology 2012250 619-625. (https://doi.org/10.1007/s00417-011-1863-x)

12 Eckstein AK, Finkenrath A, Heiligenhaus A, Renzing-Köhler K, Esser J, Krüger C, Quadbeck B, Steuhl KP \& Gieseler RK. Dry eye syndrome in thyroid-associated ophthalmopathy: lacrimal expression of TSH receptor suggests involvement of TSHR-specific autoantibodies. Acta Ophthalmologica Scandinavica 200482 291-297. (https://doi. org/10.1111/j.1395-3907.2004.00268.x)

13 Yi W. Expression of intercellular adhesion molecule-1 in lacrimal glands derived from thyroid associated ophthalmopathy. Chinese Ophthalmic Research 200321 153-155.

14 Mourits MP, Prummel MF, Wiersinga WM \& Koornneef L. Clinical activity score as a guide in the management of patients with Graves' ophthalmopathy. Clinical Endocrinology 199747 9-14. (https://doi. org/10.1046/j.1365-2265.1997.2331047.x)

15 Bingham CM, Harris MA, Realini T, Nguyen J, Hogg JP \& SivakCallcott JA. Calculated computed tomography volumes of lacrimal glands and comparison to clinical findings in patients with thyroid eye disease. Ophthalmic Plastic and Reconstructive Surgery 201430 116-118. (https://doi.org/10.1097/IOP.0000000000000015)

16 Byun JS, Moon NJ \& Lee JK. Quantitative analysis of orbital soft tissues on computed tomography to assess the activity of thyroidassociated orbitopathy. Graefe's Archive for Clinical and Experimental Ophthalmology 2017255 413-420. (https://doi.org/10.1007/s00417016-3538-0)

17 Parmar H \& Ibrahim M. Extrathyroidal manifestations of thyroid disease: thyroid ophthalmopathy. Neuroimaging Clinics of North America 200818 527-536, viii. (https://doi.org/10.1016/j.nic.2008.03.003)

18 Gagliardo C, Radellini S, Morreale Bubella R, Falanga G, Richiusa P, Vadalà M, Ciresi A, Midiri M \& Giordano C. Lacrimal gland herniation in Graves ophthalmopathy: a simple and useful MRI biomarker of disease activity. European Radiology 202030 2138-2141. (https://doi. org/10.1007/s00330-019-06570-5)
19 Ercan MT \& Unlenen E. Accumulation of some small molecular weight complexes of 99mTc in experimental abscesses. Nuclear Medicine and Biology 199421 143-149. (https://doi.org/10.1016/09698051(94)90002-7)

20 Ujhelyi B, Erdei A, Galuska L, Varga J, Szabados L, Balazs E, Bodor M, Cseke B, Karanyi Z, Leovey A, et al. Retrobulbar 99mTc-diethylenetriamine-pentaacetic-acid uptake may predict the effectiveness of immunosuppressive therapy in Graves' ophthalmopathy. Thyroid 200919 375-380. (https://doi.org/10.1089/ thy.2008.0298)

21 Yang M, Chung Y, Lang S, Yawata N, Seah LL \& Looi A. The tear cytokine profile in patients with active Graves' orbitopathy. Endocrine 201859 402-409. (https://doi.org/10.1007/s12020-017-1467-2)

22 Tamboli DA, Harris MA, Hogg JP, Realini T \& Sivak-Callcott JA. Computed tomography dimensions of the lacrimal gland in normal Caucasian orbits. Ophthalmic Plastic and Reconstructive Surgery 201127 453-456. (https://doi.org/10.1097/IOP.0b013e31821e9f5d)

23 Bahn RS. Graves' ophthalmopathy. New England Journal of Medicine 2010362 726-738. (https://doi.org/10.1056/NEJMra0905750)

24 Conrady CD, Joos ZP \& Patel BCK. Review: the lacrimal gland and its role in dry eye. Journal of Ophthalmology 20162016 7542929. (https:// doi.org/10.1155/2016/7542929)

25 Harris MA, Realini T, Hogg JP \& Sivak-Callcott JA. CT dimensions of the lacrimal gland in Graves orbitopathy. Ophthalmic Plastic and Reconstructive Surgery 201228 69-72. (https://doi.org/10.1097/ IOP.0b013e31823c4a3a)

26 Lee JS, Lee H, Kim JW, Chang M, Park M \& Baek S. Computed tomographic dimensions of the lacrimal gland in healthy orbits. Journal of Craniofacial Surgery 201324 712-715. (https://doi. org/10.1097/SCS.0b013e31827fecc0)

27 Galuska L, Leovey A, Szucs-Farkas Z, Garai I, Szabo J, Varga J \& Nagy EV. SPECT using 99mTc-DTPA for the assessment of disease activity in Graves' ophthalmopathy: a comparison with the results from MRI. Nuclear Medicine Communications 200223 1211-1216. (https://doi. org/10.1097/00006231-200212000-00010)

28 Galuska L, Varga J, Szucs Farkas Z, Garai I, Boda J, Szabo J, Leovey A \& Nagy EV. Active retrobulbar inflammation in Graves' ophthalmopathy visualized by Tc-99m DTPA SPECT. Clinical Nuclear Medicine 200328 515-516. (https://doi.org/10.1097/01.RLU.0000067520.87766.B6)

29 Jiang C, Li X, Zhao M, Dend H, Huang J, Liu D \& Xu X. Efficacy of 99mTc-DTPA orbital SPECT/CT on the evaluation of lacrimal gland inflammation in patients with thyroid associated ophthalmopathy. Zhong Nan Da Xue Xue Bao Yi Xue Ban 201944 322-328. (https://doi. org/10.11817/j.issn.1672-7347.2019.03.014)

30 Huh HD, Kim JH, Kim SJ, Yoo JM \& Seo SW. The change of lacrimal gland volume in Korean patients with thyroid-associated ophthalmopathy. Korean Journal of Ophthalmology 201630 319-325. (https://doi.org/10.3341/kjo.2016.30.5.319)

Received in final form 18 December 2021

Accepted 11 January 2022

Accepted Manuscript published online 11 January 2022 https://ec.bioscientifica.com https://doi.org/10.1530/EC-21-0590 (c) 2022 The authors Published by Bioscientifica Ltc

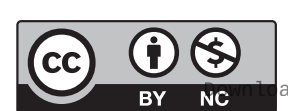

This work is licensed under a Creative Commons Attribution-NonCommercial 4.0 International License. ded from Bioscientifica.com at 04/26/2023 02:20:11PM 